\title{
AIDS, Clinical Trials on Minorities
}

National Cancer Institute

\section{Source}

National Cancer Institute. AIDS, Clinical Trials on Minorities. NCI Thesaurus. Code C15765.

AIDS clinical trials related to individuals of non-Caucasian descent. 